\title{
Translational Shape Invariance and the Inherent Potential Algebra
}

\author{
Asim Gangopadhyaya \\ Loyola University Chicago, agangop@luc.edu \\ Jeffrey Mallow \\ Loyola University Chicago, jmallow@luc.edu \\ Uday P. Sukhatne \\ University of Illinois at Chicago, sukhatme@uic.edu
}

Follow this and additional works at: https://ecommons.luc.edu/physics_facpubs

Part of the Physics Commons

\section{Recommended Citation}

Gangopadhyaya, Asim, Jeffry V. Mallow, and Uday P. Sukhatme. "Translational Shape Invariance and the Inherent Potential Algebra." Physical Review A 58, no. 6 (December 1, 1998): 4287-92. doi:10.1103/ PhysRevA.58.4287.

This Article is brought to you for free and open access by the Faculty Publications and Other Works by Department at Loyola eCommons. It has been accepted for inclusion in Physics: Faculty Publications and Other Works by an authorized administrator of Loyola eCommons. For more information, please contact ecommons@luc.edu.

\section{(c) (i) $\Theta$}

This work is licensed under a Creative Commons Attribution-Noncommercial-No Derivative Works 3.0 License. (C) 1999 The American Physical Society. 


\title{
Translational shape invariance and the inherent potential algebra
}

\author{
Asim Gangopadhyaya, ${ }^{1, *}$ Jeffry V. Mallow, ${ }^{1, \dagger}$ and Uday P. Sukhatme ${ }^{2, \$}$ \\ ${ }^{1}$ Department of Physics, Loyola University Chicago, Chicago, Illinois 60626 \\ ${ }^{2}$ Department of Physics, University of Illinois at Chicago (m/c 273), 845 West Taylor Street, Chicago, Illinois 60607-7059
}

(Received 8 May 1998)

\begin{abstract}
For all quantum-mechanical potentials that are known to be exactly solvable, there are two different, and seemingly independent methods of solution. The first approach is the potential algebra of symmetry groups; the second is supersymmetric quantum mechanics, applied to shape-invariant potentials, which comprise the set of known exactly solvable potentials. Using the underlying algebraic structures of Natanzon potentials, of which the translational shape-invariant potentials are a special subset, we demonstrate the equivalence of the two methods of solution. In addition, we show that, while the algebra for the general Natanzon potential is so $(2,2)$, the subgroup so(2,1) suffices for the shape invariant subset. Finally, we show that the known set of exactly solvable potentials in fact constitutes the full set of such potentials. [S1050-2947(98)09611-5]

PACS number(s): 03.65.Fd, 03.65.Sq, 02.20.-a
\end{abstract}

\section{INTRODUCTION}

Natanzon potentials [1] form a complete set of exactly solvable potentials of nonrelativistic quantum mechanics for which the Schrödinger equation reduces to the hypergeometric equation. In Refs. [2,3], Alhassid and co-workers have studied the group structure of these quantum-mechanical systems, related their Hamiltonians to the Casimir operator of an underlying so $(2,2)$ algebra, and determined all their quantum states by group-theoretical methods.

In supersymmetric quantum mechanics (SUSY-QM) [4] one applies a different algebraic method. The exactly solvable problems in SUSY-QM are described by superpotentials $W(x, a)$ that obey a special integrability condition,

$$
W^{2}\left(x, a_{0}\right)+\frac{d W\left(x, a_{0}\right)}{d x}=W^{2}\left(x, a_{1}\right)-\frac{d W\left(x, a_{1}\right)}{d x}+R\left(a_{0}\right)
$$

known as shape invariance [5,6]. $R\left(a_{0}\right)$ is a constant and the parameter $a_{1}$ is a function of $a_{0}$; i.e., $a_{1}=f\left(a_{0}\right)$. For a shape-invariant system, the entire spectrum can be determined algebraically by a procedure similar to that of the one-dimensional harmonic oscillator, without ever referring to the underlying differential equations. Although most of the known shape-invariant potentials (SIP) belong to the Natanzon class, there are a few exceptions [7,8].

In a previous work [9], we have shown that problems for which (1) there is a translational change of parameters $a_{1}$ $=f\left(a_{0}\right)=a_{0}+$ const and (2) $R\left(a_{0}\right)$ is a linear function of $a_{0}$, the shape-invariance condition of Eq. (1) implies the presence of an so $(2,1)$ dynamical algebra. Hence these problems are solvable by either method. As shown in Ref. [4], potentials of this type include Morse, Scarf I, Scarf II, and

\footnotetext{
*Electronic address: agangop@luc.edu, asim@uic.edu

†Electronic address: jmallow@1uc.edu

\#Electronic address: sukhatme@uic.edu
}

generalized Pöschl-Teller. However, the formalism used in Ref. [9] is not readily extendable to other shape-invariant potentials.

In this paper, we generalize our work of Ref. [9]. However, we have used a different approach here that is closely based on the work of Alhassid, Gürsey, and Iachello [2]. The authors of Ref. [2] have shown that a Hamiltonian with a general Natanzon potential has an so(2,2) symmetry. We study here the algebra of Natanzon potentials that are also shape invariant. We find that general Natanzon potentials when subjected to a further constraint give the entire set of shape-invariant potentials. The shape-invariant potentials that reduce to the confluent hypergeometric equation can be obtained as a limit [10]. We also find that while the algebra for the general Natanzon potential is so(2,2), a subgroup so $(2,1)$ suffices for all the shape-invariant problems of the Natanzon type.

Thus, this paper connects all the shape-invariant potentials of translational type $\left(a_{1}=a_{0}+\right.$ const $)$ to an algebraic structure that has many interesting consequences. Some time ago it was discovered that spectra of potentials with translational shape invariance can be exactly determined by the supersymmetric WKB method [11], which usually only gives approximate results. The reason for this exactness was very puzzling. However, in light of this group-theoretical connection, this result may not be that difficult to understand, as various authors have demonstrated the exactness of WKB results on a group manifold [12].

In Sec. II, we will quickly review the formalism of SUSY-QM and shape invariance. In Sec. III, we will briefly describe our previous work [9] where we connected a subset of SIP's to so $(2,1)$ potential algebra. In Sec. IV, we discuss the potential algebra of a general Natanzon potential. This section will closely follow Ref. [2]. In Sec. V, we identify conditions under which the general Natanzon potential reduces to a shape-invariant potential. We then show that this condition has a finite number of solutions for shape-invariant potentials; however, they generate all the known shapeinvariant potentials of translational type. 


\section{SUSY-QM AND SHAPE INVARIANCE}

In this section, we very briefly describe supersymmetric quantum mechanics (SUSY-QM), and also show how SUSY-QM with shape invariance allows one to completely determine the spectrum of a quantum system. For a more detailed description, see Ref. [4].

A quantum-mechanical system given by a potential $V_{-}(x)$ can alternatively be described by its ground-state wave function $\psi_{0}^{(-)}$. From the Schrödinger equation for the ground-state wave function $\left[-\psi_{0}^{\prime \prime}+V(x) \psi_{0}=0\right]$, it follows that the potential can be written as $V_{-}(x)=\left(\psi_{0}^{\prime \prime} / \psi_{0}\right)$, where the prime denotes differentiation with respect to $x$. (It is assumed that the potential is properly adjusted to make the ground-state energy $E_{0}=0$.) In SUSY-QM, it is customary to express the system in terms of the superpotential $W(x)$ $=-\left(\psi_{0}^{\prime} / \psi_{0}\right)$. The ground-state wave function is then given by $\psi_{0} \sim \exp \left[-\int_{x_{0}}^{x} W(x) d x\right] ; x_{0}$ is an arbitrarily chosen reference point. The Hamiltonian $H_{-}$can now be written as

$$
\begin{aligned}
H_{-} & =\left(-\frac{d^{2}}{d x^{2}}+V_{-}(x)\right)=\left(-\frac{d^{2}}{d x^{2}}+W^{2}(x)-\frac{d W(x)}{d x}\right) \\
& =\left(-\frac{d}{d x}+W(x)\right)\left(\frac{d}{d x}+W(x)\right) .
\end{aligned}
$$

(We are using units with $\hbar$ and $2 m=1$.) In analogy with the harmonic-oscillator raising and lowering operators, we have introduced two operators: $A=[(d / d x)+W(x)]$, and its Hermitian conjugate $A^{+}=[-(d / d x)+W(x)]$. Thus $H_{-}$ $=A^{\dagger} A$.

However, one can easily construct another Hermitian operator $H_{+}=A A^{\dagger}$ and show that the eigenstates of $H_{+}$are isospectral with excited states of $H_{-}$. The Hamiltonian $H_{+}$, with potential $V_{+}(x)=\left\{W^{2}(x)+[d W(x)] / d x\right\}$, is called the superpartner of the Hamiltonian $H_{-}$. To show the isospectrality mentioned above, let us denote the eigenfunctions of $H_{ \pm}$that correspond to eigenvalues $E_{n}^{ \pm}$, by $\psi_{n}^{( \pm)}$. For $n$ $=1,2, \ldots$,

$$
\begin{aligned}
H_{+}\left(A \psi_{n}^{(-)}\right) & =A A^{+}\left(A \psi_{n}^{(-)}\right)=A\left(A^{+} A \psi_{n}^{(-)}\right) \\
& =A H_{-}\left(\psi_{n}^{(-)}\right)=E_{n}^{-}\left(A \psi_{n}^{(-)}\right) .
\end{aligned}
$$

Hence, except for the ground state that obeys $A \psi_{0}^{(-)}=0$, for all other states $\psi_{n}^{(-)}$of $H_{-}$there exists a state $\psi_{n-1}^{(+)}$ $\propto A \psi_{n}^{(-)}$of $H_{+}$with exactly the same energy, i.e., $E_{n}^{+}$ $=E_{n+1}^{-}$, where $n=0,1,2, \ldots$. Conversely, one also has $A^{+} \psi_{n}^{(+)} \propto \psi_{n+1}^{(-)}$.

Thus, if the eigenvalues and the eigenfunctions of $H_{-}$ were known, one would automatically obtain the eigenvalues and the eigenfunctions of $H_{+}$, which is in general a completely different Hamiltonian.

Now, let us consider the special case where $V_{-}\left(x, a_{0}\right)$ is a shape-invariant potential. For such systems, potentials $V_{+}\left(x, a_{0}\right)=V_{-}\left(x, a_{1}\right)+R\left(a_{0}\right)$. Their superpotential $W$ obeys the integrability condition of Eq. (1). Since potentials $V_{+}\left(x, a_{0}\right)$ and $V_{-}\left(x, a_{1}\right)$ differ only in an additive constant, their common ground-state wave function is given by $\psi_{0}^{(-)}\left(x, a_{1}\right) \sim \exp \left[-\int_{x_{0}}^{x} W\left(x, a_{1}\right) d x\right]$. [The ground-state energy of $H_{+}\left(x, a_{0}\right)$ is $R\left(a_{0}\right)$, because the ground-state energy of $V_{-}\left(x, a_{1}\right)$ vanishes.] Now using SUSY-QM algebra, the first excited state of $H_{-}\left(x, a_{0}\right)$ is given by $A^{+}\left(x, a_{0}\right) \psi_{0}^{(-)}\left(x, a_{1}\right)$ and the corresponding eigenvalue is $R\left(a_{0}\right)$. By iterating this procedure, the $(n+1)$ th excited state is given by

$$
\psi_{n+1}^{(-)}\left(x, a_{0}\right) \sim A^{+}\left(a_{0}\right) A^{+}\left(a_{1}\right) \cdots A^{+}\left(a_{n}\right) \psi_{0}^{(-)}\left(x, a_{n}\right),
$$

and corresponding eigenvalues are given by

$$
E_{0}^{(-)}=0 ; \quad \text { and } \quad E_{n}^{(-)}=\sum_{k=0}^{n-1} R\left(a_{k}\right) \quad \text { for } n>0
$$

[To avoid notational complexity, we have suppressed the $x$ dependence of operators $A\left(x, a_{0}\right)$ and $A^{+}\left(x, a_{0}\right)$. $]$ Thus, for a shape-invariant potential, one can obtain the entire spectrum of $H_{-}$by the algebraic methods of SUSY-QM.

Most of the known exactly solvable problems possess a spectrum generating algebra (SGA) $[2,3,13]$. We would like to find out if there is any connection between SGA and shape invariance of these systems. As we shall see later, the type of SGA that is most relevant to us is known as potential algebra, studied extensively by Alhassid and co-workers [2,3]. In potential algebra, the Hamiltonian of the system is written in terms of the Casimir operator $\left(C_{2}\right)$ of the algebra, and the energy of states specified by an eigenvalue $\omega$ of $C_{2}$ is fixed. Different states with fixed $\omega$ represent eigenstates of a set of Hamiltonians that differ only in values of parameters and share a common energy. For a system with an so(2,1) potential algebra, the different values of parameters are eigenvalues of operator $J_{3}$, chosen to form a complete set of commuting observables. This is very similar to the case of shapeinvariant potentials. In the next section, we will attempt to establish this connection in a more concrete fashion. In fact, for a set of solvable quantum-mechanical systems we shall explicitly show that shape invariance leads to a potential algebra.

\section{SHAPE INVARIANCE AND CONNECTION TO ALGEBRA}

Let us consider a generic shape-invariant potential $V_{-}\left(x, a_{0}\right)$ with a translational change of parameters $a_{m+1}$ $=a_{m}+\delta=a_{0}+(m+1) \delta$, where $\delta$ is a constant. For the superpotential $W\left(x, a_{m}\right) \equiv W(x, m)$, the shape-invariance condition is

$$
W^{2}(x, m)+W^{\prime}(x, m)=W^{2}(x, m+1)-W^{\prime}(x, m+1)+R(m) .
$$

It is natural to ask whether the change of parameters can be formally accomplished by the action of a ladder-type operator. With this in mind, we define an operator $J_{3}=-i \partial / \partial \phi$ $\equiv-i \partial_{\phi}$, analogous to the $z$ component of the angularmomentum operator. It acts upon functions in the space described by two coordinates $x$ and $\phi$, and its eigenvalues $m$ will play the role of the parameter of the potential. We also define two more operators, $J^{-}$and its Hermitian conjugate $J^{+}$by 


$$
J^{ \pm}=e^{ \pm i \phi}\left[ \pm \frac{\partial}{\partial x}-W\left(x,-i \partial_{\phi} \pm \frac{1}{2}\right)\right] .
$$

The factors $e^{ \pm i \phi}$ in $J^{ \pm}$ensure that they indeed behave as ladder operators for the quantum number $m$. (The factor $\pm \frac{1}{2}$ merely "symmetrizes" the $J^{ \pm}$'s.) Operators $J^{ \pm}$are basically of the same form as the $A^{ \pm}$operators of SUSY-QM, except that the parameter $m$ of the superpotential $W$ is replaced by operators $\left(J_{3} \pm \frac{1}{2}\right)$. Explicit computation shows that

$$
\left[J_{3}, J^{ \pm}\right]= \pm J^{ \pm}
$$

and hence operators $J^{ \pm}$change the eigenvalues of the $J_{3}$ operator by unity, similar to the ladder operators of angular momentum [so(3)]. Now let us determine the commutator $\left[J^{+}, J^{-}\right]$:

$$
\begin{aligned}
{\left[J^{+}, J^{-}\right]=} & J^{+} J^{-}-J^{-} J^{+} \\
= & {\left[-\frac{\partial^{2}}{\partial x^{2}}+W^{2}\left(x, J_{3}-\frac{1}{2}\right)-W^{\prime}\left(x, J_{3}-\frac{1}{2}\right)\right] } \\
& -\left[-\frac{\partial^{2}}{\partial x^{2}}+W^{2}\left(x, J_{3}+\frac{1}{2}\right)+W^{\prime}\left(x, J_{3}+\frac{1}{2}\right)\right] \\
= & -R\left(J_{3}+\frac{1}{2}\right),
\end{aligned}
$$

where we have used the shape-invariance condition (4). Thus, we see that shape invariance enables us to close the algebra of $J_{3}$ and $J^{ \pm}$to

$$
\left[J_{3}, J^{ \pm}\right]= \pm J^{ \pm}, \quad\left[J^{+}, J^{-}\right]=-R\left(J_{3}+\frac{1}{2}\right) .
$$

Now, if the function $R\left(J_{3}\right)$ is linear in $J_{3}$, the algebra of Eq. (8) reduces to that of $\operatorname{so}(3)$ or $\operatorname{so}(2,1)$ [9]. Several SIP's are of this type, among them are the Morse, Scarf I, Scarf II, and generalized Pöschl-Teller potentials. For these potentials, $R\left(J_{3}+\frac{1}{2}\right)=2 J_{3}[4]$, and Eq. (8) reduces to an $\operatorname{so}(2,1)$ algebra and hence establishes a connection between shape invariance and potential algebra. With a slightly different formalism, Balantekin arrived at a similar conclusion for these SIP's [14]. However, there are many other important systems like Coulomb, Eckart, etc. where $R\left(a_{0}\right)$ is not linear in $a_{0}$, and these cases will be discussed later.

\section{DIFFERENTIAL REALIZATION OF so(2,2)}

Before establishing a connection between a general $\mathrm{Na}$ tanzon Hamiltonian and an so(2,2) potential algebra, we will discuss a realization of so $(2,2)$ algebra in terms of differential operators on a $(2,2)$-hyperboloid. For consistency, we use the formalism and the notations of Refs. [2,3].

A (2,2)-hyperboloid is defined by

$$
\begin{aligned}
& x_{1}=\rho \cosh \chi \cos \phi, \quad x_{2}=\rho \cosh \chi \sin \phi, \\
& x_{3}=\rho \sinh \chi \cos \theta, \quad x_{4}=\rho \sinh \chi \sin \theta,
\end{aligned}
$$

where $\phi$ and $\theta$ are rotation angles in the $x_{1}, x_{2}$ and $x_{3}, x_{4}$ planes, respectively $[0 \leqslant \phi, \theta<2 \pi]$. Six generators of the algebra, $J_{i}$ and $K_{i}(i=1, \ldots, 3)$ can be chosen as

$$
\begin{gathered}
J_{1}=\left(x_{2} p_{3}+x_{3} p_{2}\right), \quad J_{2}=-\left(x_{1} p_{3}+x_{3} p_{1}\right), \\
J_{3}=\left(x_{1} p_{2}-x_{2} p_{1}\right), \\
K_{1}=\left(x_{1} p_{4}+x_{4} p_{1}\right), \quad K_{2}=\left(x_{2} p_{4}+x_{4} p_{2}\right), \\
K_{3}=\left(x_{3} p_{4}-x_{4} p_{3}\right) .
\end{gathered}
$$

Operators $p_{i}$ represent derivatives $-i\left(\partial / \partial x_{i}\right)$. The algebraic relations obeyed by these operators are given by

$$
\begin{array}{ccc}
{\left[J_{1}, J_{2}\right]=-i J_{3},} & {\left[J_{2}, J_{3}\right]=i J_{1},} & {\left[J_{3}, J_{1}\right]=i J_{2},} \\
{\left[K_{1}, K_{2}\right]=-i J_{3},} & {\left[K_{2}, K_{3}\right]=i J_{1},} & {\left[K_{3}, K_{1}\right]=i J_{2},} \\
{\left[J_{1}, K_{2}\right]=-i K_{3},} & {\left[J_{2}, K_{3}\right]=i K_{1},} & {\left[J_{3}, K_{1}\right]=i K_{2},} \\
{\left[K_{1}, J_{2}\right]=-i K_{3},} & {\left[K_{2}, J_{3}\right]=i K_{1},} & {\left[K_{3}, J_{1}\right]=i K_{2} .}
\end{array}
$$

The above algebra can be decomposed in terms of two commuting so $(2,1)$ algebras generated by

$$
A_{i}=\frac{1}{2}\left(J_{i}+K_{i}\right), \quad B_{i}=\frac{1}{2}\left(J_{i}-K_{i}\right) .
$$

These operators commute; i.e., $\left[A_{i}, B_{j}\right]=0$. Using Eqs. (9) and (11), the differential realization can be written explicitly as $[2,3]$

$$
\begin{aligned}
& A^{ \pm} \equiv A_{1} \pm i A_{2}=\frac{1}{2} e^{ \pm i(\phi+\theta)} \times\left[\mp \frac{\partial}{\partial \chi}+\tanh \chi\left(-i \partial_{\phi}\right)+\operatorname{coth} \chi\left(-i \partial_{\theta}\right)\right], \\
& A_{3}=-\frac{i}{2}\left(\partial_{\phi}+\partial_{\theta}\right), \\
& B^{ \pm \equiv} B_{1} \pm i B_{2}=\frac{1}{2} e^{ \pm i(\phi-\theta)} \\
& \times\left[\mp \frac{\partial}{\partial \chi}+\tanh \chi\left(-i \partial_{\phi}\right)+\operatorname{coth} \chi\left(+i \partial_{\theta}\right)\right], \\
& B_{3}=-\frac{i}{2}\left(\partial_{\phi}-\partial_{\theta}\right) .
\end{aligned}
$$

The so $(2,1)$ algebra obeyed by these operators is

$$
\left[A_{3}, A^{ \pm}\right]= \pm A^{ \pm}, \quad\left[A^{+}, A^{-}\right]=-2 A_{3}
$$

and a similar one for the $B$ 's. The Casimir operator $C_{2}$ is given by

$$
\begin{aligned}
C_{2}= & 2\left(A_{3}^{2}-A_{+} A_{-}-A_{3}\right)+2\left(B_{3}^{2}-B_{+} B_{-}-B_{3}\right) \\
= & {\left[\frac{\partial^{2}}{\partial \chi^{2}}+(\tanh \chi+\operatorname{coth} \chi) \frac{\partial}{\partial \chi}+\operatorname{sech}^{2} \chi\left(-i \partial_{\phi}\right)^{2}\right.} \\
& \left.-\operatorname{cosech}^{2} \chi\left(-i \partial_{\theta}\right)^{2}\right] .
\end{aligned}
$$

Operators $A_{3}, B_{3}$, and $C_{2}$ can be simultaneously diagonalized, and their actions on their common eigenstates are given by 


$$
\begin{gathered}
C_{2}\left|\omega, m_{1}, m_{2}\right\rangle=\omega(\omega+2)\left|\omega, m_{1}, m_{2}\right\rangle, \\
A_{3}\left|\omega, m_{1}, m_{2}\right\rangle=m_{1}\left|\omega, m_{1}, m_{2}\right\rangle, \\
B_{3}\left|\omega, m_{1}, m_{2}\right\rangle=m_{2}\left|\omega, m_{1}, m_{2}\right\rangle .
\end{gathered}
$$

It is worth mentioning at this point that the Casimir operator given above is indeed self-adjoint with respect to a measure $\sinh \chi \cosh \chi d \chi d \phi d \theta$.

\section{NATANZON POTENTIALS}

The Schrödinger equation for any Natanzon potential can be reduced by a point canonical transformation (a general similarity transformation followed by an appropriate change of independent variable) $[10,15,16]$ to the hypergeometric equation. A general potential $U(r)$ of the Natanzon type is implicitly defined by [1]

$$
U[z(r)]=\frac{-f z(1-z)+h_{0}(1-z)+h_{1} z}{R(z)}-\frac{1}{2}\{z, r\},
$$

where $R(z)=a z^{2}+b_{0} z+c_{0}=a(1-z)^{2}-b_{1}(1-z)+c_{1}$ and $f, h_{0}, h_{1}, a, b_{0}, b_{1}, c_{0}, c_{1}$ are constants. The Schwarzian derivative $\{z, r\}$ is defined by

$$
\{z, r\} \equiv \frac{d^{3} z / d r^{3}}{d z / d r}-\frac{3}{2}\left[\frac{d^{2} z / d r^{2}}{d z / d r}\right]^{2}
$$

The relationship between variables $z(0<z<1)$ and $r$ is implicitly given by

$$
\left(\frac{d z}{d r}\right)=\frac{2 z(1-z)}{\sqrt{R(z)}}
$$

To avoid a singularity in $U(z(r))$, one assumes that $R(z)$ has no singularity in the domain $(0,1)$. The Schrödinger equation is given by

$$
\left[\frac{d^{2}}{d r^{2}}+\left\{\left(\frac{d z}{d r}\right)^{2} I(z)+\frac{1}{2}\{z, r\}\right\}\right]=0,
$$

where

$$
I(z)=\frac{\left(1-\lambda_{0}^{2}\right)(1-z)+\left(1-\lambda_{1}^{2}\right) z+\left(\mu^{2}-1\right) z(1-z)}{4 z^{2}(1-z)^{2}}
$$

and

$$
\begin{aligned}
\left(1-\mu^{2}\right)= & a E-f, \quad\left(1-\lambda_{0}^{2}\right)=c_{0} E-h_{0}, \\
& \left(1-\lambda_{1}^{2}\right)=c_{1} E-h_{1} .
\end{aligned}
$$

To connect the Casimir operator $C_{2}$ of the so(2,2) algebra [Eq. (14)] to the general Natanzon potential, we will first perform a similarity transformation on $C_{2}$ by a function $F$ and then follow that up by an appropriate change of variable $\chi \rightarrow g(r)$. Under the similarity transformation,

$$
\begin{gathered}
\frac{d}{d \chi} \rightarrow F \frac{d}{d \chi} F^{-1}=\left(\frac{d}{d \chi}-\frac{\dot{F}}{F}\right), \\
\frac{d^{2}}{d \chi^{2}} \rightarrow\left(\frac{d^{2}}{d \chi^{2}}-\frac{2 \dot{F}}{F} \frac{d}{d \chi}+\frac{2 \dot{F}^{2}}{F^{2}}-\frac{\ddot{F}}{F}\right),
\end{gathered}
$$

where dots represent derivatives with respect to $\chi$. The Casimir operator $C_{2}$ of Eq. (14) transforms as

$$
\begin{aligned}
C_{2} \rightarrow \widetilde{C}_{2}= & {\left[\frac{d^{2}}{d \chi^{2}}+\left(\tanh \chi+\operatorname{coth} \chi-\frac{2 \dot{F}}{F}\right) \frac{d}{d \chi}\right.} \\
& +\frac{2 \dot{F}^{2}}{F^{2}}-\frac{\ddot{F}}{F}-(\tanh \chi+\operatorname{coth} \chi) \frac{\dot{F}}{F} \\
& \left.+\operatorname{sech}^{2} \chi\left(-i \partial_{\phi}\right)^{2}-\operatorname{cosech}^{2} \chi\left(-i \partial_{\theta}\right)^{2}\right] .
\end{aligned}
$$

Now, let us carry out a change of variable from $\chi$ to $r$ via $\chi=g(r)$. We are going to denote differentiation with respect to $r$ by a prime. Operators $d / d \chi$ and $d^{2} / d \chi^{2}$ transform as

$$
\frac{d}{d \chi}=\frac{1}{g^{\prime}} \frac{d}{d r}, \quad \frac{d^{2}}{d \chi^{2}}=\frac{1}{g^{\prime 2}}\left[\frac{d^{2}}{d r^{2}}-\frac{g^{\prime \prime}}{g^{\prime}} \frac{d}{d r}\right] .
$$

The operator $\widetilde{C}_{2}$ now transforms into

$$
\begin{aligned}
\widetilde{C}_{2}= & \frac{1}{g^{\prime 2}}\left[\frac{d^{2}}{d r^{2}}+\left\{-\frac{g^{\prime \prime}}{g^{\prime}}-\frac{2 F^{\prime \prime}}{F}+g^{\prime}(\tanh g+\operatorname{coth} g)\right\}\right. \\
& \times \frac{d}{d r}+\frac{2 F^{\prime 2}}{F^{2}}-\frac{F^{\prime \prime}}{F}+\frac{F^{\prime} g^{\prime \prime}}{F g^{\prime}}-\frac{F^{\prime} g^{\prime}}{F}(\tanh g+\operatorname{coth} g) \\
& \left.+g^{\prime 2}\left(\operatorname{sech}^{2} g\left(-i \partial_{\phi}\right)^{2}-\operatorname{cosech}^{2} g\left(-i \partial_{\theta}\right)^{2}\right)\right]
\end{aligned}
$$

In order for $g^{\prime 2} \widetilde{C}_{2}$ to be a Schrödinger Hamiltonian, we require the expression inside the curly brackets in Eq. (21) to vanish. This constrains the relationship between the two functions $F$ and $g$ to be

$$
-\frac{g^{\prime \prime}}{g^{\prime}}-\frac{2 F^{\prime}}{F}+g^{\prime}(\tanh g+\operatorname{coth} g)=0,
$$

which yields

$$
F \sim\left(\frac{\sinh (2 g)}{g^{\prime}}\right)^{1 / 2}
$$

Thus the operator $\widetilde{C}_{2}$ transforms into 


$$
\begin{aligned}
\widetilde{C}_{2}= & \frac{1}{g^{\prime 2}}\left[\frac{d^{2}}{d r^{2}}+g^{\prime 2}\left(\frac{\left(1-\tanh ^{2} g\right)^{2}-4 \tanh ^{2} g}{4 \tanh ^{2} g}\right)\right. \\
& \left.+\frac{1}{2}\{g, r\}+g^{\prime 2}\left(\operatorname{sech}^{2} g\left(-i \partial_{\phi}\right)^{2}-\operatorname{cosech}^{2} g\left(-i \partial_{\theta}\right)^{2}\right)\right] .
\end{aligned}
$$

This Casimir operator now has a form of

$$
\widetilde{C}_{2}=-\frac{1}{g^{\prime 2}} H
$$

where $H$ is a one-dimensional Hamiltonian with the potential $U(r)$ given by

$$
\begin{aligned}
E-U(r)= & g^{\prime 2}\left(\frac{\left(1-\tanh ^{2} g\right)^{2}-4 \tanh ^{2} g}{4 \tanh ^{2} g}\right)+\frac{1}{2}\{g, r\} \\
& +g^{\prime 2}\left[\operatorname{sech}^{2} g\left(-i \partial_{\phi}\right)^{2}-\operatorname{cosech}^{2} g\left(-i \partial_{\theta}\right)^{2}\right] .
\end{aligned}
$$

Now, for this potential to take the form of a general Natanzon potential, we have to relate variables $g$ and $z$ in such a way that the potential in terms of $z$ is given by Eq. (16). Since the potential has to be a ratio of two quadratic functions of $z$, this is accomplished with the identification $z$ $=\tanh ^{2} g$, which leads to

$$
\begin{aligned}
U(z(r)) & =\frac{E R+\left[-\frac{7}{4}+\frac{5}{2} z-\frac{7}{4} z^{2}\right]-z(1-z)\left(-i \partial_{\phi}\right)^{2}+(1-z)\left(-i \partial_{\theta}\right)^{2}}{R}-\frac{1}{2}\{z, r\} \\
& =\left\{-\left[a E-\frac{7}{4}+\left(-i \partial_{\phi}\right)^{2}\right] z(1-z)+\left[c_{0} E-\frac{7}{4}+\left(-i \partial_{\theta}\right)^{2}\right](1-z)+\left[\left(a+b_{0}+c_{0}\right) E-1\right]\right\} / R(z)-\frac{1}{2}\{z, r\} .
\end{aligned}
$$

We have used

$$
g^{\prime}=\frac{d g}{d z} z^{\prime}=\frac{1}{2 \sqrt{z}(1-z)} \frac{2 z(1-z)}{R}=\sqrt{\frac{z}{R}} .
$$

Now, with the following identification:

$$
\begin{gathered}
f=a E-\frac{7}{4}+\left(-i \partial_{\phi}\right)^{2}, \\
h_{0}=c_{0} E-\frac{7}{4}+\left(-i \partial_{\theta}\right)^{2}, \\
h_{1}=\left(a+b_{0}+c_{0}\right) E-1,
\end{gathered}
$$

the potential of Eq. (25) indeed has the form of a general

\begin{tabular}{|c|c|c|c|c|}
\hline$\alpha$ & $\beta$ & $z(r)$ & Superpotential & Potential \\
\hline 0 & 0 & $z=e^{-r}$ & $\tilde{m}_{1} \operatorname{coth} \frac{r}{2}+\tilde{m}_{2}$ & Eckart \\
\hline 0 & $-\frac{1}{2}$ & $z=\sin ^{2} \frac{r}{2}$ & $\tilde{m}_{1} \operatorname{cosec} r+\tilde{m}_{2} \cot r$ & $\begin{array}{l}\text { Gen. Pöschl-Teller } \\
\text { trigonometric }\end{array}$ \\
\hline 0 & -1 & $z=1-e^{-r}$ & $\tilde{m}_{1} \operatorname{coth} \frac{r}{2}+\tilde{m}_{2}$ & Eckart \\
\hline$-\frac{1}{2}$ & 0 & $z=\operatorname{sech}^{2} \frac{r}{2}$ & $\tilde{m}_{1} \operatorname{cosech} r+\widetilde{m}_{2} \operatorname{coth} r$ & Pöschl-Teller II \\
\hline$-\frac{1}{2}$ & $-\frac{1}{2}$ & $z=\tanh ^{2} \frac{r}{2}$ & $\tilde{m}_{1} \tanh \frac{r}{2}+\tilde{m}_{2} \operatorname{coth} \frac{r}{2}$ & Gen. Pöschl-Teller \\
\hline-1 & 0 & $z=1+\tanh \frac{r}{2}$ & $\tilde{m}_{1} \tanh \frac{r}{2}+\tilde{m}_{2}$ & Rosen Morse \\
\hline
\end{tabular}
Natanzon potential [Eq. (16)].

\section{SHAPE-INVARIANT NATANZON POTENTIALS FROM POTENTIAL ALGEBRA}

At this point we go back to the operators $A^{ \pm}$[Eq. (13)] and see how they transform under the similarity transformation given by $F \sim\left\{[\sinh (2 g)] / g^{\prime}\right\}^{1 / 2} \sim \sqrt{z / z^{\prime}}$. This transformation carries operators $A^{ \pm}$to

TABLE I. All allowed values of $\alpha, \beta$ and the superpotentials that they generate. Constants $\tilde{m}_{i}(i=1,2)$ are linear combinations of $m_{1}$ and $m_{2}$ of Eq. (15). Simple harmonic-oscillator, Morse, and Coulomb potentials are not included in this list, as they can be considered to be limiting cases of potentials presented here [10]. 


$$
\begin{aligned}
A^{ \pm} \rightarrow \widetilde{A}^{ \pm}=\frac{e^{ \pm i(\phi+\theta)}}{2}\left[\mp\left(\frac{d}{d \chi}+\frac{1}{2 z^{\prime}} \frac{d z^{\prime}}{d \chi}-\frac{1}{2 z} \frac{d z}{d \chi}\right)\right. \\
\left.+\tanh \chi\left(-i \partial_{\phi}\right)+\operatorname{coth} \chi\left(-i \partial_{\theta}\right)\right] .
\end{aligned}
$$

If the expression $\left[\left(1 / 2 z^{\prime}\right)\left(d z^{\prime} / d \chi\right)-(1 / 2 z)(d z / d \chi)\right]$ can be written as a linear combination of $\tanh \chi$ and coth $\chi$, operators $\widetilde{A}^{ \pm}$can be cast in a form similar to the operators $J^{ \pm}$ of Eq. (5), and the connection with shape invariance is established.

Hence to get shape-invariant potentials we require

$$
\left(\frac{1}{2 z^{\prime}} \frac{d z^{\prime}}{d \chi}-\frac{1}{2 z} \frac{d z}{d \chi}\right)=\alpha \tanh \chi+\beta \operatorname{coth} \chi .
$$

This leads to $z^{\prime}=z^{1+\beta}(1-z)^{-\alpha-\beta}$, which is another restriction on the relationship between variables $z$ and $r$. Since these variables are already constrained by Eq. (18), only a handful of solutions would be compatible with both restrictions. Thus $z(r)$ 's that are compatible with both equations are given by

$$
z^{1+\beta}(1-z)^{-\alpha-\beta}=\frac{2 z(1-z)}{\sqrt{R(z)}}
$$

where $R(z)$ is a quadratic function of $z$. After some computation, we find that there are only a finite number of values of $\alpha, \beta$ that satisfy Eq. (28). These values are listed in Table I, and they exhaust all known shape-invariant potentials that lead to the hypergeometric equation. It is also interesting to note that while the potential algebra of a general Natanzon system is so(2,2), and requires two sets of raising and lowering operators $A^{ \pm}$and $B^{ \pm}$, all known shape-invariant potentials need only one such set. For all SIP's of Table 4.1 of Ref. [4], one finds that all partner potentials are connected by a change of just one independent parameter (although other parameters which do not change are also present). Thus there is a series of potentials that only differ in one parameter. From the potential algebra perspective, all these potentials differ only by the eigenvalue of an operator that is a linear combination of $A_{3}$ and $B_{3}$, and all are characterized by a common eigenvalue of $C_{2}$. Thus, these shape-invariant potentials can be associated with an so $(2,1)$ potential algebra generated by operators $A^{+}, A^{-}$and the same linear combination of $A_{3}$ and $B_{3}$.

Note added in the proof. Recently, we became aware of work done by Cordero and Salamo [17]. In these papers, the authors show that the Natanzon potential has a spectrumgenerating algebra given by so(2,1). However, for shapeinvariant systems, the potential algebra $[2,3]$ generated by operators with a first-order derivative is more relevant. The Casimir operator of the potential algebra is essentially the same as the Hamiltonian of the shape-invariant model.

\section{ACKNOWLEDGMENTS}

A.G. acknowledges a research leave from Loyola University, Chicago, which made his involvement in this work possible. We would like to thank Professor Y. Alhassid for clarifying several points. One of us (A.G.) would also like to thank the Physics Department of the University of Illinois for their warm hospitality. Partial financial support from the U.S. Department of Energy is gratefully acknowledged.
[1] G. A. Natanzon, Theor. Math. Phys. 38, 146 (1979) [Teor. Math. Fiz. 38, 219 (1979)].

[2] Y. Alhassid, F. Gürsey, and F. Iachello, Ann. Phys. (Leipzig) 148, 346 (1983).

[3] J. Wu and Y. Alhassid, J. Math. Phys. 31, 557 (1990).

[4] F. Cooper, A. Khare, and U. Sukhatme, Phys. Rep. 251, 268 (1995), and references therein.

[5] L. Infeld and T. E. Hull, Rev. Mod. Phys. 23, 21 (1951).

[6] L. E. Gendenshtein, Pisma Zh. Eksp Teor. Fiz. 38, 299 (1983) [JETP Lett. 38, 356 (1983)].

[7] V. P. Spiridonov, Phys. Rev. Lett. 69, 398 (1992).

[8] D. Barclay, R. Dutt, A. Gangopadhyaya, A. Khare, A. Pagnamenta, and U. Sukhatme, Phys. Rev. A 48, 2786 (1993).

[9] A. Gangopadhyaya, J. V. Mallow, and U. P. Sukhatme, in Proceedings of Workshop on Supersymmetric Quantum Mechanics and Integrable Models, 1997, edited by Henrik Aratyn et al. (Springer-Verlag, Berlin, 1998).
[10] A. Gangopadhyaya, P. Panigrahi, and U. Sukhatme, Helv. Phys. Acta 67, 363 (1994).

[11] A. Comtet, A. Bandrauk, and D. K. Campbell, Phys. Lett. B 150, 381 (1985).

[12] J. S. Dowker, J. Phys. A 3, 451 (1970); Ann. Phys. (Leipzig) 62, 561 (1971); K. Funahashi, T. Kashiwa, S. Sakoda, and K. Fujii, J. Math. Phys. 36, 3232 (1995); 36, 4590 (1995).

[13] A. O. Barut, A. Inomata, and R. Wilson, J. Phys. A 20, 4075 (1987); 20, 4083 (1987); M. J. Englefield, J. Math. Phys. 28, 827 (1987); M. J. Englefield and C. Quesne, J. Phys. A 24, 3557 (1987); R. D. Tangerman and J. A. Tjon, Phys. Rev. A 48, 1089 (1993).

[14] A. B. Balantekin, Phys. Rev. A 57, 4188 (1998).

[15] F. Cooper, J. N. Ginnocchio, and A. Wipf, J. Phys. A 22, 3707 (1989).

[16] R. De, R. Dutt, and U. Sukhatme, J. Phys. A 25, L843 (1992).

[17] P. Cordero and S. Salamo, Found. Phys. 23, 675 (1993); J. Math Phys. 35, 3301 (1994). 